Synthesis, vol. 24 no 2, e019, diciembre 2017. ISSN 1851-779X

Universidad Nacional de La Plata.

Facultad de Humanidades y Ciencias de la Educación.

Centro de Estudios Helénicos

\title{
Ares y Dionisos: discurso político y poesía trágica en Fenicias de Eurípides
}

\section{Brenda López Saiz* \\ * Universidad de Chile, Chile}

Cita sugerida: López Saiz, B. (2017). Ares y Dionisos: discurso político y poesía trágica en Fenicias de Eurípides. Synthesis, 24 (2), e019. https://doi.org/10.24215/1851779Xe019 


\title{
Ares y Dionisos: discurso político y poesía trágica en Fenicias de Eurípides
}

\author{
Brenda López Saiz
}

Universidad de Chile, Chile

\section{Resumen:}

En este trabajo, proponemos que en Fenicias de Eurípides se realiza una reflexión meta-dramática a partir de la oposición entre dos campos semánticos: por una parte, la guerra y la discordia, el poder político, el interés individual y el discurso público asociado a prácticas "sofísticas"; por otra, la paz y la concordia entre los miembros de la comunidad, los vínculos de philía, el bien colectivo y la mousiké coral, objeto a su vez de pasajes auto-referenciales. A través de ella, nos parece que la obra se plantea críticamente ante la situación política y bélica que Atenas enfrenta alrededor de 411-409 a.C -periodo en que se sitúa con mayor probabilidad su representación- y, a la vez, realiza una reflexión acerca de la función social de la poesía trágica, oponiéndola a las nuevas formas discursivas que, codificadas y enseñadas por los “sofistas", se imponen en el ámbito público ateniense. 1

Palabras Clave: Eurípides, Meta-drama, Sofística, Auto-referencialidad coral, Proyección coral.

\section{ABStRACT:}

In this paper, we read Euripides' Phoenissae as a metadramatic reflection which is developed through the opposition of two semantic fields: on the one hand, war and eris, political power, individual interest and public discourse associated to "sophistic" practices; on the other hand, peace and concord among members of the community; philia bonds, colective well-being and choral mousike, which in turn are subject of self-referential passages. Through this opposition, we propose that the play poses a critique to the political and military situation faced by Athens between 411 and 409 b.C, and, at the same time, it develops a meta-dramatic reflection about the social function of tragic poetry, opposing it to the new kinds of discourses which, taught by the sophists, prevail in Athenian public sphere.

KEYWORDS: Euripides, Meta-drama, Sophistic, Choral self-reference, Choral projection.

Fenicias fue muy probablemente representada entre los años 411-409 a.C., ${ }^{2}$ es decir, en un período complejo para la política ateniense: en el plano externo, el desastre de Sicilia a fines de 413 da paso a la reactivación de una ofensiva espartana que gana terreno tanto en el propio territorio del Ática como entre las póleis jonias. Paralelamente, en el ámbito interno, Atenas vive la preparación e imposición de un gobierno oligárquico durante $411 \mathrm{y}$, si bien dicho gobierno tiene una duración muy breve, su desarrollo revela la pugna descarnada por el poder como práctica distintiva de los líderes del periodo, tal como lo conocemos a través del relato de Tucídides. En la obra, se elabora dramáticamente un conocido episodio del mito de los Labdácidas: el asalto a Tebas liderado por Polinices, quien ataca la ciudad junto con el contingente argivo reivindicando su derecho a gobernar, derecho usurpado por su hermano Eteocles, al mando de la ciudad tebana. En lo que es una tendencia característica de la producción tardía de Eurípides, tal como ha sido puesto en evidencia por diversos críticos, ${ }^{3}$ la obra posee una estructura dramática compleja: incluye un gran número de figuras y perspectivas dramáticas (doce en total), su argumento se desarrolla a través de una sucesión no lineal de las acciones y sucesos, ${ }^{4}$ hace un uso intensivo de la intertextualidad y contiene una serie de elementos que pueden ser considerados como estrategias de carácter meta-dramático y auto-reflexivo.

Entre estas últimas, se cuenta la utilización de dos procedimientos vinculados al coro, denominados por Henrichs en dos conocidos artículos como "auto-referencialidad coral" y "proyección coral”. El primero, refiere a "the self-description of the tragic chorus as performer of choreia" (1995: 57); el segundo, corolario del anterior, a los pasajes en que los coros trágicos "locate their own dancing in the past or the future, in contrast 
to the here and now of their immediate performance, or when choruses project their collective identity onto groups of dancers distant from the concrete space of the orchestra and dancing in the allusive realm of the dramatic imagination" (1996: 49). ${ }^{5}$ En dichos pasajes, para Henrichs, el coro asume una doble dimensión: por una parte, los comentarios auto-referenciales aluden a la posición de la figura colectiva representada por el coro en la propia situación dramática; por otra, traen a primer plano la condición del coro como componente central de las Fiestas Dionisíacas realizadas en Atenas. La doble connotación dramática y extra-dramática del coro, para el autor, se establece a partir de la función ritual que sería común a ambas dimensiones, siendo su función enfatizar dicho carácter del coro y la tragedia en tanto parte de la celebración pública en honor a Dionisos.

El coro de Fenicias constituye un claro ejemplo de los dos procedimientos auto-reflexivos señalados por Henrichs: en la obra, el grupo de doncellas fenicias en tanto figura dramática es en sí mismo un coro, pues ellas están destinadas a rendir culto a Apolo mediante el canto y la danza en Delfos, destino final del viaje que tiene a Tebas como parada intermedia; sin embargo, el ejercicio de dicha función se ve diferido e indefinidamente pospuesto debido a la situación crítica que el grupo de jóvenes doncellas encuentra en Tebas, diferimiento que se expresa a través de la estructura dramática misma: mientras el final de la obra cierra la acción concerniente a la familia de los Labdácidas y a la ciudad de Tebas, el destino de las doncellas fenicias no recibe una resolución. Debido a esta situación dramática, la "auto-referencialidad" y la "proyección coral" se presentan de manera simultánea: cuando el coro hace alusión a su propia actividad de coreutas destinadas a Apolo, inevitablemente refiere a una actividad que se proyecta hacia un futuro avizorado desde el precario e incierto presente. De esa manera, la auto-referencialidad y la proyección coral no se dan únicamente en pasajes u odas específicas -tal como ocurre en otras tragedias de Eurípides y también de Sófocles-, sino en la propia situación dramática del coro.

La función y el sentido de dichos procedimientos se despliegan, por una parte, al interior de la acción dramática: mediante el contraste, ellos enfatizan el desorden y el horror de la situación vivida en Tebas, la cual hace imposible el rito coral y la relación ordenada con los dioses que este supone. $\mathrm{Al}$ mismo tiempo, tal como fue planteado por Henrichs, el sentido de la auto-referencialidad es también de índole auto-reflexiva, esto es, reflexiona sobre la especificidad de la poesía trágica y sobre su lugar en la sociedad ateniense. No obstante, a diferencia de lo propuesto por el crítico, en este artículo proponemos que esa reflexión no refiere a la dimensión ritual de la actividad poético-coral, en tanto vinculada al culto público y cívico de Dionisos, sino que tiene como objeto la función social que la poesía coral ejerció tradicionalmente y aquella que la poesía trágica puede ejercer en el contexto político de fines del siglo $\mathrm{V}$, marcado por la crisis y la desintegración moral y política de la sociedad ateniense. Asimismo, proponemos que dicha reflexión no se efectúa únicamente mediante la auto-referencialidad y la proyección coral, sino mediante la asociación de la práctica coral-poética y ritual, a la que el coro auto-refiere, con un conjunto de valores que, en virtud de su mención reiterada, asumen el carácter de motivos dramáticos: la paz, la lealtad y el respeto a los vínculos de philía y el bien de la colectividad. Dichos motivos son, a su vez, recurrentes en los discursos de Yocasta, lo que la integra con el coro en un campo semántico que, a su vez, se configura a partir de la oposición a aquel compuesto por los motivos de la discordia (Eris), la guerra (Ares), el poder político y el privilegio del interés individual, todos ellos asociados también a una práctica discursiva particular, la erística, y encarnados en las figuras dramáticas de Eteocles y Polinices.

\section{LOS INTERESES Y DISCURSOS DE LA DISCORDIA}

Fenicias desarrolla una sucesión de acciones dramáticas en torno a la espera del ataque argivo a Tebas. A través de esa acción militar, Polinices espera acceder al gobierno de la ciudad apoyado por su suegro Adrasto, rey de Argos, en respuesta a la violación del pacto que sellaba la sucesión rotativa de ambos hermanos en el poder. Al igual que versiones anteriores del mito de los Labdácidas, tales como Siete contra Tebas de Esquilo y 
Antígona y Edipo Rey de Sófocles -a las que probablemente podemos agregar la Tebaida-, la acción de ambos hermanos, que amenaza con destruir a la estirpe y a la ciudad de Tebas, se presenta como parte de la maldición que asola a la familia en distintos pasajes: el discurso de Yocasta en el prólogo (vv. 1-87); menciones del coro de fenicias en el segundo y tercer stásimon (vv. 811-13; 1019-1054), y la rhêsis de Antígona en el éxodos (vv. 1495-1507). ${ }^{6}$

Esa dimensión mítica que inscribe las acciones de los Labdácidas en la maldición familiar, tal como ocurre en Esquilo, podría atenuar la individualidad de las figuras dramáticas y, sobre todo, el ejercicio de su voluntad en las acciones que emprenden, toda vez que estas son determinadas por fuerzas superiores al control humano. Sin embargo, a mi parecer, la determinación mítico-divina de los sucesos es relativizada a través de la configuración de los personajes Eteocles y Polinices, la cual se efectúa en el primer episodio mediante un diálogo entre Polinices y Yocasta, y un debate discursivo que integra elementos del contexto ateniense de la época: a través de él, los personajes míticos asumen cualidades que los acercan a los individuos y prácticas propias de la sociedad ateniense del periodo y, en consecuencia, lejos de ser sujetos zarandeados por la necesidad ineluctable, son exhibidos como individuos en cuyas manos, intereses y decisiones reside el destino de la colectividad, en lo que puede ser interpretado como comentario a los agentes y sucesos de su contexto.

Son dos los aspectos del contexto inmediato que inciden en la conformación dramática individual de ambas figuras: la similitud con prácticas y actitudes políticas que encontramos referidas en otras fuentes de la época, principalmente en Tucídides, y la asociación con la práctica discursiva y con la reflexión sobre el lenguaje atribuida a los "sofistas".

En Fenicias, a diferencia de Siete contra Tebas, Polinices es una figura dramática, cuya voz y punto de vista complejizan la presentación de las acciones y sucesos. En la tragedia de Esquilo, él es el agresor, cuyas acciones fuera de escena generan la situación central de la obra, esto es, la representación de la amenaza que el ataque supone para la ciudad, ante la cual Eteocles aparece como el gobernante abocado a defenderla. En la versión de Eurípides, en cambio, la inclusión de Polinices da pie a la presentación del gobierno de la pólis como una actividad en la que no entra en juego la defensa irrestricta de la comunidad, concebida como valor supremo y absoluto, sino una diversidad de intereses individuales, cuya afirmación y realización no solo no garantiza el bien colectivo, sino que incluso entra en pugna con este.

La presentación del punto de vista de Polinices se realiza en dos escenas del extenso primer episodio, cuando este ingresa a Tebas y a escena convocado por su madre, quien promueve un encuentro entre ambos hermanos, con la esperanza de encontrar una solución pacífica al conflicto. En la primera escena, su entrada furtiva y atemorizada da lugar al encuentro con el coro y con su madre, con quien desarrolla un extenso diálogo cuyo tema central es la experiencia del exilio. En la segunda, ambos hermanos se enfrentan en un debate en el que el planteamiento de sus respectivas posiciones concluye con la afirmación de la inevitabilidad del enfrentamiento armado.

En esa presentación de la situación y posición de Polinices, encontramos elementos que aparecen reiteradamente en casos del acontecer político del periodo narrados por Tucídides, entre los cuales el ejemplo más conspicuo es el de la figura de Alcibíades. Al igual que este y muchos otros líderes atenienses, Polinices ha sido exiliado por razones políticas; a raíz de ello, emprende acciones contra la propia ciudad en alianza con una pólis enemiga; ante esa situación abiertamente contraria a su comunidad, que desde el sentido común lo transforma en un traidor, insiste no obstante en la ambigua compatibilidad entre sus acciones y el amor a la patria.

En la escena del encuentro entre Yocasta y Polinices, asistimos a la exposición del sufrimiento suscitado por el exilio, experiencia que se presenta como un conjunto de privaciones de los distintos aspectos que configuran

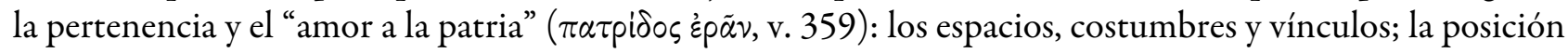
económica y social, y la posibilidad de participar libremente en las decisiones políticas, la cual anacrónica y significativamente se designa como libertad de expresión ( $\pi \alpha p p \eta \sigma i \alpha)$, en sintonía con el contexto democrático 
extra-dramático (vv. 366-370; 389-395; 400-407). Así, en lo que Polinices denomina como "amor a la patria", predomina un vínculo con la pólis basado en aspectos individuales y personales, actitud que se mantiene en su decisión de atacar Tebas en pos de la reivindicación de su derecho a gobernar. Lo restringido de su definición y posición, que desconsidera la dimensión colectiva de la patria, cuyo fundamento es a la vez institucional y religioso, es puesto en evidencia por Yocasta, quien lo amonesta en los siguientes términos:

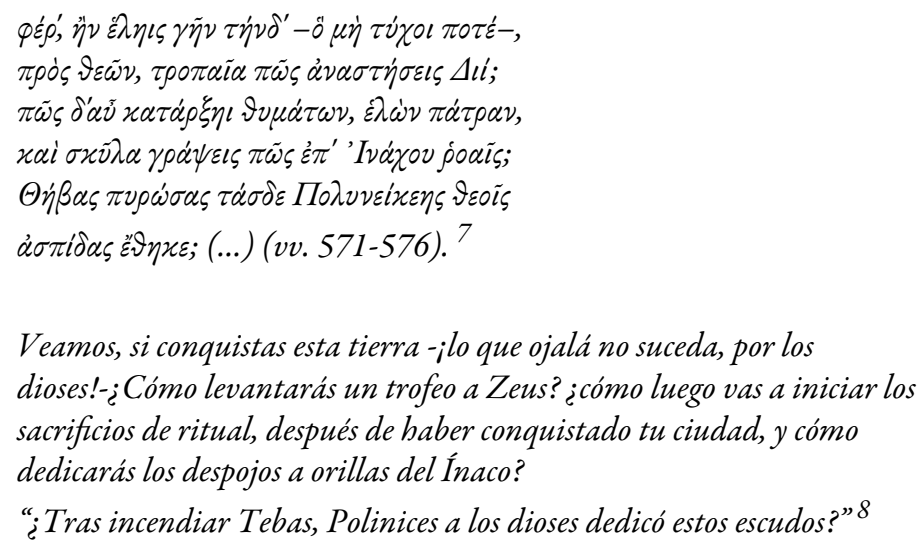

Una visión semejante de la relación con la patria, se presenta de manera elocuente en el discurso de Alcibíades ante los espartanos, en el cual trata de generar confianza mediante un hábil discurso que justamente redefine el amor a la patria a partir de una posición individualista:

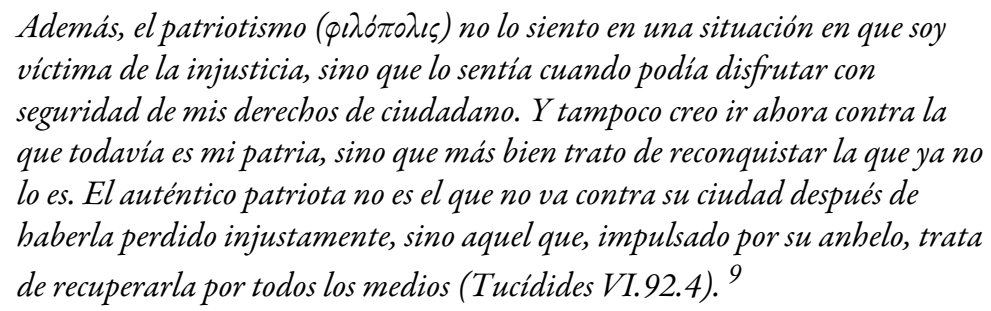

Las posibles analogías con el contexto de representación de Fenicias continúan en el enfrentamiento entre Eteocles y Polinices mediante un debate, en el cual se aprecian tanto ideas y prácticas atribuidas a la 'sofística', como tendencias vigentes en la actividad política del contexto. Ambos hermanos inician sus respectivas argumentaciones problematizando el propio discurso a partir de la relación entre los nombres y sus referentes (ónoma/prâgma), remitiendo así tanto a un tema de discusión central entre pensadores de la época que desde Platón pasaron a ser agrupados y conocidos como 'sofistas', como a un tópico recurrente en los debates públicos. En primer lugar, Polinices afirma la veracidad de su discurso a partir de la oposición que asocia el discurso falso a la injusticia, la enfermedad y la ornamentación, y el 'verdadero' a la justicia y la sencillez, en lo que constituye también un lugar común reiterado en textos de la época, ya sea para criticar lo que ha pasado a ser conocido como 'retórica sofística', como es el caso de comedias de Aristófanes -en particular Nubes y Ranas-; para plantear una determinada visión del lenguaje, tal como la encontramos en el Encomio a Helena de Gorgias; o bien como recurso de la argumentación política, tal como se aprecia, por ejemplo, en el debate sobre Mitilene entre Cleón y Diódoto relatado por Tucídides (III.37-48). ${ }^{10} \mathrm{Al}$ igual que en dicho debate, dichas afirmaciones resultan irónicas si consideramos, como plantea Mastronarde, que Polinices "delivers a speech which in structure and particular effects is strikingly skilful rhetoric", ${ }^{11}$ aun cuando, a diferencia de Cleón, Polinices no pareciera usar deliberada y cínicamente dichas ideas como estrategia retórica, sino más bien ser portador de uno de los discursos vigentes en el contexto de representación de la tragedia: 


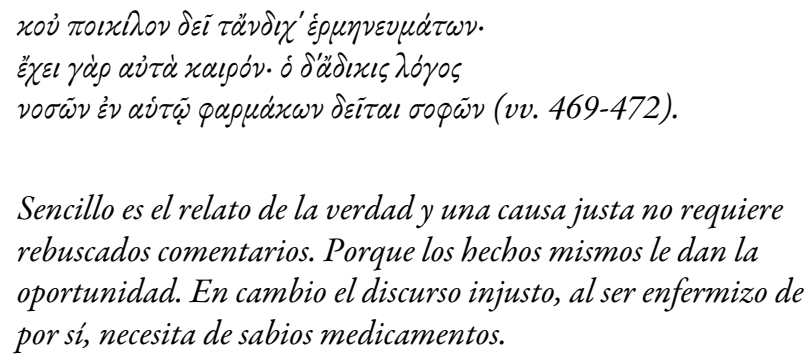

A dicho planteamiento, Eteocles se opone a través de una argumentación que evoca ideas de Protágoras y Gorgias en función de un discurso político y moral de índole relativista. Mientras Polinices defiende la existencia de una visión verdadera y justa, Eteocles echa mano de la tesis del 'homo mensura' atribuida a Protágoras, afirmando la existencia de percepciones distintas de un mismo fenómeno: no a todos les parece

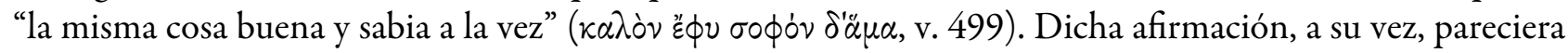
vincularse a la noción de antilogía, según la cual para todo enunciado u objeto pueden sostenerse predicados

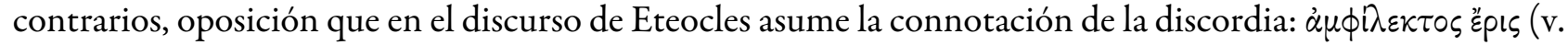
500). De esa manera, el relativismo implicado en la idea de que el hombre es la medida de todas las cosas, junto a la concepción antilógica del conocimiento y el lenguaje, son utilizadas como herramientas para legitimar la discordia y la contienda como forma de reaccionar ante el desacuerdo. Más aún, para reforzar esa postura que busca su fundamento argumentativo en el relativismo, Eteocles no se contenta con proponer una visión antilógica del discurso y la percepción, sino que, a partir de las ideas gorgianas que postulan una separación radical entre lógos y referente, ${ }^{12}$ niega la posibilidad de existencia de ideas y valores comunes que puedan ser designados por un significante unívoco:

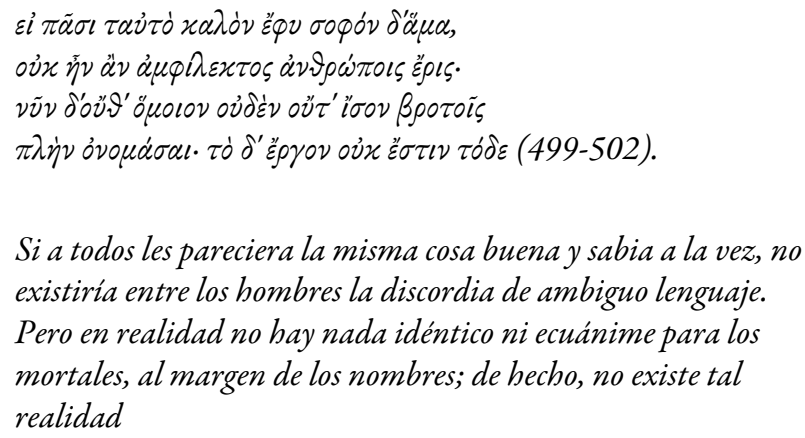

Así, tal como propone Mastronarde, "both the language and content of Et.'s speech are meant to associate Et. with the clever young men who used the training of the sophists to discomfit their traditionally minded elders and to justify selfish and agressive behaviour". ${ }^{13}$ En sintonía con esa práctica, desde su introducción el discurso de Eteocles se presenta como ejemplo de la erística, es decir, la práctica discursiva cuya finalidad es obtener la victoria argumentativa sobre el oponente, sin consideración por la verdad y haciendo uso de los recursos que sean necesarios para obtenerla. ${ }^{14}$ En este caso, la victoria buscada es el rechazo a la reivindicación de Polinices, que implicaría compartir el poder ejerciéndolo alternadamente, rechazo que a su vez implica la aceptación de una confrontación armada, desconsiderando tanto el vínculo de philía y los intereses del oîkos y el génos, como el bien de la colectividad. Así, los argumentos de Eteocles se amoldan a la defensa de su interés $\mathrm{y}$, en último término, se revelan como un simulacro inconducente, dado que las diferencias se juegan en el terreno de la fuerza: Eteocles es el tirano que ejerce el poder y no está dispuesto a cederlo. Ante ello, tanto el sofisticado relativismo que cuestiona la validez absoluta de la posición de Polinices, como el argumento de que no es posible llegar ahora a un acuerdo mediante la palabra, toda vez que Polinices ha llegado a Tebas al mando de un ejército, se revelan como irónicamente falaces. 
Al declarar el ejercicio del poder como su objetivo, y el uso de la fuerza como vía legítima para mantenerlo, Eteocles manifiesta así explícitamente lo que, en el relato de Tucídides, es la real pero encubierta motivación de la acción política de los líderes atenienses en su oportunista adhesión a una u otra forma de gobierno. $\mathrm{Al}$ referirse al alejamiento de líderes de la oligarquía de los cuatrocientos por un régimen de los cinco mil, comenta lo siguiente:

Pero eso era un pretexto político esgrimido de palabra, y la mayor parte de ellos adoptaba aquella postura por ambiciones personales, siguiendo un camino en el que por lo general encuentra su perdición una oligarquía nacida de una democracia, puesto que desde el primer día no todos no sólo no pretenden ser iguales, sino que cada uno aspira a ser el primero con mucha diferencia(...) (Tucídides. VIII.89.3-4).

Así, tanto en Fenicias como en Tucídides, el ejercicio del poder político se presenta como producto de un juego de fuerzas que enfrenta a individuos en pugna por la supremacía personal, en el que la violencia es un medio legítimo para adquirirla, y el discurso una herramienta subordinada a esta. En el enfrentamiento entre ambos hermanos, entonces, lejos de ser una vía racional de resolución de los conflictos y de dilucidación de la mejor opción, el discurso y el debate argumentativo se presentan como instrumento explícito de la afirmación del poder, fundado en un relativismo moral y discursivo, que no solo no impide, sino legitima la violencia y la fuerza.

\section{MOUSIKÉ Y CONCORDIA}

A diferencia de lo que observamos en la presentación de Eteocles y Polinices, las figuras de Yocasta y el coro de fenicias se asocian constantemente a actitudes y valores que emanan del vínculo de philía, el mismo que resultó irrelevante para ambos hermanos. Por una parte, y a diferencia de la obra de Sófocles, Yocasta no se define primordialmente por su cualidad de mujer incestuosa, sino por su condición de madre que padece por la suerte de sus hijos, intenta reparar la discordia y, ante su muerte, elige morir junto a ellos. Por otra, el coro de fenicias, ligado a Tebas a partir del tronco común de los Agenóridas, comparte el sufrimiento de sus lejanos parientes, en una adhesión que contrasta tanto con ambos hermanos, como con la actitud de Creonte al final de la obra: mientras los miembros cercanos de la familia se destruyen, las parientes extranjeras cumplen con la lealtad y la compasión que la philía supone.

En ellas, a su vez, el motivo de la philía se asocia al uso de otros tipos de discurso, que se contraponen al uso de la erística por parte de los hermanos. En su intento de evitar la guerra a través de un encuentro entre ambos, Yocasta representa la confianza en la palabra argumentativa y persuasiva como vía de resolución de los conflictos en pos del bien común, tanto de la familia como de la pólis, confianza esta en la que resuena el optimismo que encontramos, por ejemplo, en Euménides de Esquilo. Asimismo, su propia argumentación se centra en valores que esa visión del discurso supone, la equidad y la mesura, valores que, como vimos, la actitud de ambos hermanos también desecha.

Por otra parte, tanto Yocasta como sobre todo el coro se presentan asociadas a la mousiké. En el caso de la primera, la asociación con el canto y la danza se produce justamente en el momento de su reencuentro con Polinices, en una monodia que hace evidente la conexión entre mousiké, philía y emoción. Ante su alegría, Yocasta hace explícita la dificultad de encontrar palabras adecuadas que la expresen y, en seguida, la manifiesta danzando alrededor de Polinices y describiendo su acción en los siguientes términos:

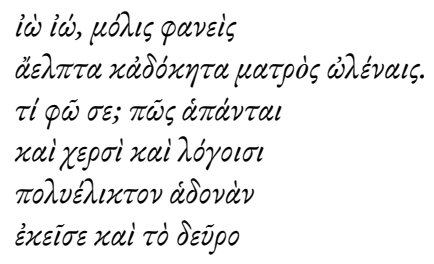




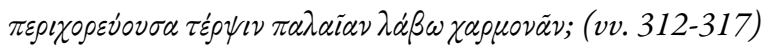

¡Ay! ; Ay! A duras penas hete aquil contra toda previsión y de modo inesperado entre los brazos de tu madre./ ¿Qué voy a decirte? ¿Cómo puedo, por todos los medios,/ con mis manos y con palabras, / danzando a tu alrededor/ a uno y otro lado/con giros que me dan placer, recoger el gozo de mi antigua felicidad?

Ya en el caso del coro, la mousiké es la actividad que constituye una de sus características definitorias en tanto figura dramática pues, como dijimos, este coro representa a su vez a un grupo de doncellas destinadas a rendir culto a Apolo en Delfos a través de la choreía. Es decir, representa a un grupo de personas destinadas a ejercer la función social realizada tradicionalmente por la lírica coral en Grecia, en la que la poesía, el canto y la danza son una práctica que contribuye a generar y reafirmar la identidad y la cohesión social, en torno a ritos y prácticas que requieren a su vez la acción conjunta y cohesionada de un grupo. ${ }^{15} \mathrm{Al}$ mismo tiempo, en un nivel meta-dramático, dicha condición alude también a la práctica coral en tanto actividad central de las Dionisíacas, tanto en la competencia de ditirambos como en la representación de los dramas de las que este coro es un ejemplo.

Ahora bien, la obra plantea desde un inicio una oposición entre esa práctica de índole ritual, a la que el grupo de fenicias está destinada, y la guerra causada por la discordia entre ambos hermanos, situación que por una parte difiere su avance hasta su destino final y que, por otra, alude simbólicamente a una sociedad en discordia y descomposición, en la que la unidad, la cohesión y la armonía que la mousiké representan no es posible.

Dicha oposición se esboza en la párodos, en la que la anticipación y el anhelo del coro por la función que ha de cumplir en un futuro incierto se contraponen a su situación presente, y en la que el espacio de Tebas es caracterizado en contraste con Delfos. En esa oda, el espacio de Delfos es descrito a través de una serie de elementos naturales y de lugares que constituyen la morada de la divinidad: en las primeras estrofa y antístrofa, Delfos es descrito como el lugar bajo la sagrada cima nevada del Parnaso en el que Apolo reside, donde la fuente Castalia espera a las doncellas para la purificación ritual necesaria para servir al dios. Ya en la estrofa siguiente (epodo interno), una extensa descripción es desarrollada bajo la forma de una invocación, en la que Delfos aparece como el lugar en que Apolo comparte espacio con Dionisos, asociando a ese dios con la fiesta, el vino y la fertilidad, y a ambas divinidades con la danza coral. Ese lugar divino es caracterizado también con los atributos de la luz, claridad y altura, los cuales, junto a la forma de la invocación, enfatizan su carácter de manifestación de un reino intemporal y permanente, localizado sobre la realidad humana perecedera.

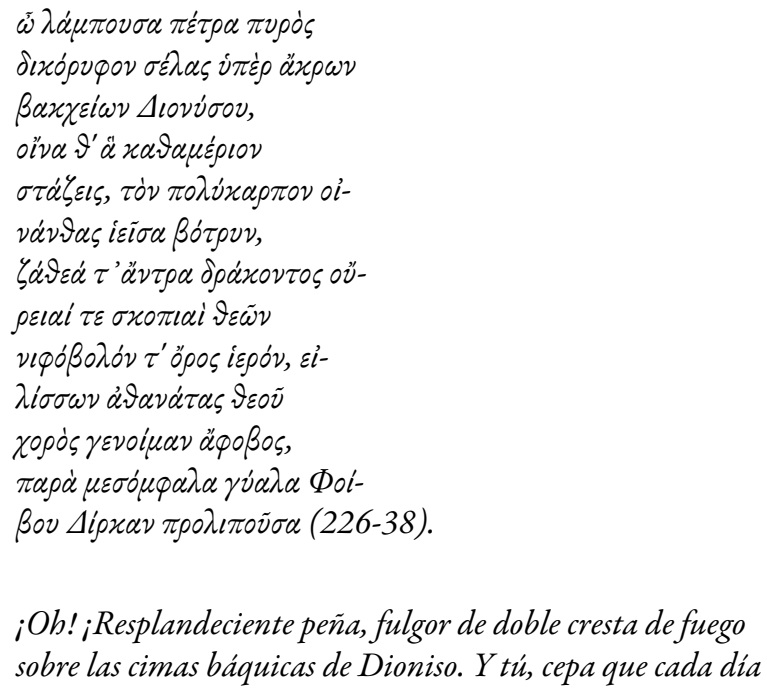




\begin{abstract}
derramas, en continua eclosión la uva arracimada, divinas cavernas del dragón y montaraces atalayas de los dioses, y sagrada montaña que cubre la nieve! Ojalá que, libre de temores llegue a formar el coro que da vueltas en torno del dios inmortal, junto a las cavidades del ombligo terrestre, en dominios de Febo, dejando atrás la fontana de Dirce.
\end{abstract}

Tebas, en cambio, es descrita en la segunda estrofa y antístrofa como el lugar de Ares, caracterizado por el fuego destructivo que difunde una luz sombría sobre la ciudad; por inminente sangre, lucha y destrucción, causadas por la discordia en la familia real. Esa oposición esbozada en la párodos, prefigura la caracterización de Tebas en el segundo stásimon, en el que la ciudad asediada por Ares se define justamente por la ausencia de coros en honor a Dionisos:

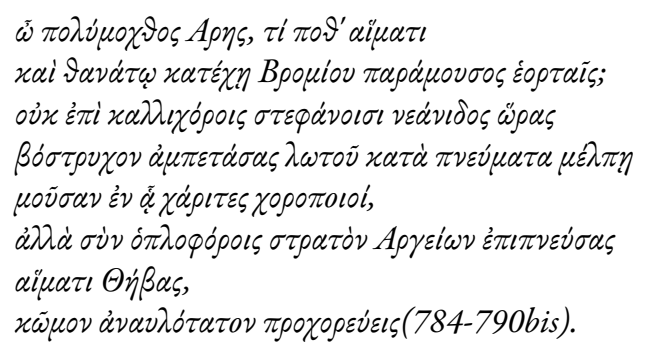

De esa manera, en la primera parte de la obra se construye una oposición semántica entre un mundo en que la relación ordenada y gozosa entre hombres y dioses se asocia a la práctica coral ritual (Delfos), y una pólis marcada por el conflicto, la destrucción, la ausencia de mousiké y la presencia devastadora de un solo dios, Ares. Como vimos con anterioridad, ese espacio se asocia también a un uso particular de la palabra y, al mismo tiempo, posee claras connotaciones extra-dramáticas que lo vinculan al contexto ateniense. Teniendo esto en consideración, podemos entonces postular que la auto-referencialidad coral, por una parte, puede ser leída como un comentario acerca de la situación de Atenas en el periodo: la ausencia de choreía y la imposibilidad de cantar que afecta a este coro en particular representan la discordia, violencia y desorden que imperan en la pólis. Por otra, la presentación de esta Tebas-Atenas como un espacio en el que la disputa por el poder y el beneficio individual es asociada a una particular forma de discurso público, y a la ausencia de mousiké, puede ser leída como una reflexión acerca del rol de la poesía en la sociedad. Este coro auto-reflexivo puede ser considerado entonces como una representación de las funciones social, religiosa y educativa que la lírica coral ejerció tradicionalmente en la sociedad griega. En consecuencia, su asociación en la obra con el mundo ordenado y pacífico representado por Delfos, en contraste con la pólis dominada por la discordia en actos y palabras, puede ser interpretada como una reflexión acerca de la crisis cultural y política vivida por Atenas en ese momento, en la que el menoscabo del rol ejercido por la lírica coral constituiría no solo una consecuencia, sino también una causa de la crisis. A partir de esa asociación, se podría adjudicar al coro una perspectiva autorial, portadora de una visión nostálgica de la cohesión social representada por la mousiké. A mi juicio, sin embargo, la perspectiva autorial no radica en un punto de vista particular -en este caso el del coro-, sino en la decisión que integra y hace interactuar las distintas perspectivas y discursos que hemos examinado al 
interior de una estructura dramática. Su introducción en la obra, ligándolo a los motivos de la philía y la paz, y oponiéndolo a la guerra, la discordia y el discurso político tal como está siendo usado en la Atenas contemporánea, sirve para dramatizar la crisis: en este momento, en que los coros simbólicamente no son más posibles, la poesía trágica asume entonces el rol crítico de representar la descomposición de la sociedad. Sin embargo, así como la llegada del coro a Delfos, la posibilidad de la concordia queda indefinidamente diferida.

\section{REFERENCIAS}

Eurípides (1998). Fenicias. Introducción, traducción y notas de García Gual, C., en Tragedias, vol. III,. Madrid. Kovacs, D. (2002). Euripides: Helen, Phoenician Women, Orestes, Cambridge, Mass.

Mastronarde, D. (2004). Euripides: Phoenissa,Cambridge.

Tucídides (2007) Historia de la guerra del Peloponeso,Traducción y notas de Juan José Torres Esbarranch, Madrid. Dunn, F. (1996). Tragedy's End: Closure and Innovation in Euripidean Drama, Oxford.

Henrichs, A. (1995) "Why should I dance? Choral self-referentiality in Greek tragedy”, Arion 3.1: 56-111.

Henrichs, A. (1996) "Dancing in Athens, Dancing on Delos: Some Patterns of Choral Projection in Euripides", Philologus 140: 48-62.

Kerferd, G. B. (1981). The Sophistic Movement, Cambridge.

Kowalzig, B. (2004) "Changing Choral Worlds: Song-Dance and Society in Athens and Beyond”, en P. Murray \& P. Wilson Music and the Muses: the Culture of Mousike in the Classical Athenian City, Oxford: 39-66.

Mastronarde, D. (2010). The Art of Euripides: Dramatic Technique and Social Context, Cambridge.

Michelini, A. (2009) "The 'packed-full' drama in late Euripides: Phoenissae", en J.R.C. Cousland \& J. R. Hume, The Play of Texts and Fragments: Essays in Honour of Martin Cropp,Leiden, Boston: 169-182.

Pfister, M. (1988). The Theory and Analysis of Drama, Cambridge.

Wohl, V. (2015). Euripides and the Politics of Form, Princeton, Oxford.

\section{NoTAS}

1 Este trabajo se inscribe en el proyecto Fondecyt de Iniciación $\mathrm{N}^{\circ} 11140911$, dedicado al estudio de la producción tardía de Eurípides.

2 Cf. Mastronarde (2004: 11-14), Kovacs (2002: 203).

3 Cf. Dunn (1996), Michelini (2009), Mastronarde (1994, 2010), Wohl (2015), entre otros.

$4 \quad$ Utilizamos las definiciones de acción y suceso propuestas por Pfister (1988).

5 Cf. Henrichs (1995: 57, 1996: 49).

6 En la obra, encontramos también una segunda determinación mítica: la muerte del dragón de Ares efectuada por Cadmo en la fundación de la ciudad, que según el oráculo de Tiresias requiere la muerte de Meneceo para aplacar al dios y sus efectos. No la abordamos en nuestro análisis, dado que, tal como plantea Mastronarde, dicha determinación afecta a la ciudad de Tebas, separando así el destino de la colectividad del de la estirpe de Lábdaco. Cf. Mastronarde (2004: 5). Por otra parte, nos parece que la obra presenta elementos que ponen en entredicho la validez de dicho mito en tanto historia verdadera, y por lo tanto justificación legítima del sacrificio de Meneceo. Sin embargo, no es posible desarrollar el análisis de ese tema en los límites de esta exposición.

7 Este y los siguientes pasajes citados en griego corresponden a la edición de Mastronarde (2004).

8 A lo largo del artículo, cito la traducción al español de García Gual (1998), con pocas modificaciones de mi parte.

9 Las citas de Tucídides corresponden a la traducción de Torres Esbarranch (2007).

10 Cf. Mastronarde (2004: 280-281).

11 Cf. Mastronarde (2004: 280).

12 Cf. Kerferd (1981: 81).

13 Cf. Mastronarde (2004: 288).

14 Cf. Kerferd (1981: 63).

15 Cf. Kowalzig (2004:41). 